\title{
CONFIDENCE INFORMATION SETS
}

\author{
Manish Aggarwal ${ }^{1}$ and Madasu Hanmandlu ${ }^{2}$ \\ ${ }^{I}$ IIM Ahmedabad, India \\ ${ }^{2}$ MVSR Engineering College, Hyderabad, India
}

\begin{abstract}
A new data structure for the representation of an agent's assessment of an information source value, along with the associated confidence degree is presented. The proposed structure is termed as confidence information set (CIS). It gives multiple information values, as perceived, corresponding to an information source value, along with the associated confidence degree. The usefulness of the proposed CIS in multi criteria decision making is shown through illustrative examples.
\end{abstract}

\section{KEYWORDS}

Information Set, Confusion, Imprecision, Decision Making

\section{INTRODUCTION}

The fuzzy set theory (Zadeh, 1965) represents in-exactness, ill-definedness in an information source value. However, it has its own difficulties (Aggarwal, 2016). The fuzzy concepts are the linguistic expressions, and hence limited by the words in the daily language (Zimmerman ,1983). Human thinking and feelings that conjure the uncertainty in a given situation, have certainly more comprehensions and concepts than the words in the daily language. Therefore, it is often difficult to guarantee, unequivocally, a one to one mapping between the uncertainty perceived and its depiction by means of a membership function. Moreover, a membership grade is always relative without an explicit consideration of the associated information source value. Resultantly, the interpretation of a membership grade depends on the onlooker agent. For instance, an agent's evaluation of a person as highly knowledgeable conveys only a little information and it means different for each interpreting agent. Inspired by these drawbacks of a membership function, in this work, we develop alternate structures for the representation of uncertainty.

To address this, the concept of information set is proposed in (Aggarwal,2016). It gives a linked representation of an information source value and its evaluation (termed as agent), computed through the Hanman-Anirban entropy function (Hanmandlu, 2011). Such entropy values are referred to as the information values, and collectively comprise an information set. These entropy values are easier to interpret and deal with, as shown in (Aggarwal, 2016). Since, agent is a function of a combination of parameters and an information source value, it can take many forms, including those of the commonly used membership functions like Gaussian, triangular, or trapezoidal etc.

These features of information set theory make it quite useful in any application where an agent (a DM, etc.). Besides, it is especially useful in representing vague or subjective assessment of the decision-maker (DM), in multi criteria decision making (MCDM). However, when the values are ill-defined (or vague), the agent inevitably faces confusion. That is, often ( $\mathrm{s}$ )he may have multiple possible perceptions for the same information source value. To address this, the concept of information set has been extended as hesitant information set (HIS) in (Aggarwal M, 2019), inspired by the concept of hesitant fuzzy set. A HIS gives a set of all the perceived values that an agent may have for an information source value.

HIS provides an excellent data structure to address confusion in the information set theory. However, the confusion of an agent may manifest not only in the form of multiplicity of perceived values, but also in the form of varying confidence degrees. For instance, an agent witnesses a fast-moving car. In the absence of a ready device to know the objective speed of the car, (s)he would at best try to express the perceived value for the same, but (s)he may have some confusion about the accuracy of his/her expression of the perceived speed of the car. In such a situation, the agent may find it more convenient to associate the perceived value (information value) with a degree of confidence, in place of giving multiple perceived values, as required in 
the case of HIS. This extra piece of information could be vital in the decision making application. For instance, if the agent has witnessed the car from very far distance, and therefore has a very vague idea of the real speed, he would have very low degree of confidence. In the absence of a provision to have this confidence degree of the agent, this perceived value given by the agent is very much likely to be considered as the true speed value, in contrast to what it would have been considered with a low confidence value. To address this, we are motivated to extend the concept of information set with the confidence degree of the agent. The data structure, so formed, is termed as confidence information set. The rest of the paper is organized as follows. Section 2 gives the background for the study. In Section 3, we introduce the concept of confidence information set. Section 4 concludes the paper.

\section{BACKGROUND}

\subsection{Information Set}

Let $U=\left\{u_{1}, \ldots, u_{m}\right\}$ denote the reference set with each $u_{i}$ described by a set of attributes E. The value an attribute e $\in$ E takes for $u_{i}$ is termed as information source value, and is denoted by $I_{e}\left(u_{i}\right)$. The collection of all such information source values is shown as: $I_{e}=\left\{I_{e}\left(u_{i}\right) \mid \forall u_{i} \in U\right\}$

$I_{e}$ in (1) can be seen as an information function $I_{e}: U \rightarrow I_{e}\left(u_{i}\right)$, and $I_{e}\left(u_{i}\right)$ refers to a normalized information source value in the interval $[0,1]$. The information gain function is given as:

$$
g_{e}\left(u_{i)}=e^{-\left(a_{e}\left(I_{e}\left(u_{i}\right)\right)^{3}+b_{e}\left(I_{e}\left(u_{i}\right)\right)^{2}+c_{e}\left(I_{e}\left(u_{i}\right)\right)+d_{e}\right)^{\alpha} e}\right.
$$

where $g_{e}\left(u_{i}\right)$ gives the desirability for the value that attribute e takes for alternative $u_{i}$; and $a_{e}, b_{e}, c_{e}, d_{e}$ and $\alpha_{e}$ are the free parameters, specific to e, i.e. $\left\{I_{e}\left(u_{i}\right)\right\}, \forall u_{i} \in U$. These parameters lead to different forms of $g_{-} e(\cdot)$. A set of parameters therefore uniquely represents the evaluation pattern of a DM.

The parameter values can also be the moments such as mean and standard deviation of the distribution of the information source values. This results into generation of a generalized Gaussian function. For instance, let $a_{e}=0, b_{e}=0, c_{e}=\frac{1}{\sigma x}, d_{e}=-\frac{m_{e}}{\sigma_{e}}\left(\right.$ where $m_{e}$ refers to the mean value $\left.\frac{1}{n} \Sigma_{i} I_{e}\left(u_{i}\right)\right)$, then we obtain $g_{e}(\cdot)$ as:

$$
g_{e}\left(u_{i}\right)=e^{-}\left(\frac{I_{e}\left(u_{i}\right)-m_{e}}{\sigma_{e}}\right)^{\alpha_{e}}
$$

where, $\alpha_{\mathrm{e}}$ is termed as a DM's utilitarian parameter for attribute e which uniquely specifies a DM's evaluation behaviour. Different values of $\propto_{e}=0.5,1, \ldots, 5$ correspond to different shapes of $g_{e}=2$ shown in Figure 1. A Gaussian function is obtained at $\alpha_{\mathrm{e}}=2$.

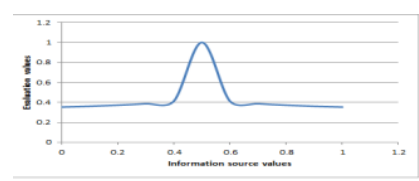

(a) $\alpha=0.1$

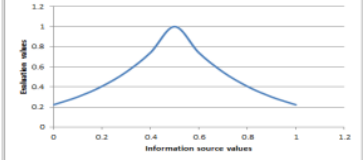

(b) $\alpha=1.0$

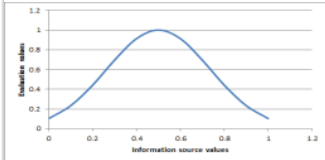

(c) $\alpha=2.0$

Figure 1. The evaluation values of the form $g_{e}\left(u_{i}\right)$, as obtained with information gain function, shown in (3)

The uncertainty in $\left\{I_{e}\left(u_{i}\right)\right\}, \forall u_{i} \in U$, as evaluated by $g_{e}(\cdot)$ is given as: $Q_{e}=\sum I_{e}\left(u_{i}\right) g_{e}\left(u_{i}\right)$

where $I_{e}\left(u_{i}\right) g_{e}\left(u_{i}\right)$ gives $I_{e}\left(u_{i}\right)$, as perceived by the agent. The set $\left\{I_{e}\left(u_{i}\right) g_{e}\left(u_{i}\right)\right\}$ constitutes an information set $\mathcal{S}_{e}$, shown as: $\mathcal{S}_{e}=\left\{I_{e}\left(u_{i}\right) g_{e}\left(u_{i}\right)\right\}, \forall u_{i} \in U$

Each element of $\mathcal{S}_{e}$ is expressed as: $\mathrm{S}_{\mathrm{e}}\left(u_{i}\right)=I_{e}\left(u_{i}\right) g_{e}\left(u_{i}\right)$ (6), which is an entropy (information) value.

\section{CONFIDENCE INFORMATION SET}

The information set is generalized as confidence information set (CIS) by having the confidence degrees associated with each of the information values in the information set. A confidence information set Cover soft universe (U,C), with $\Theta$ as a vector of the confidence values, is a set defined by a mapping $F: C \rightarrow \mathcal{S} \times \mathbb{R}$, and $\ominus: C \rightarrow \mathbb{R}$. The confidence information set is formed from the sets of ordered pairs of 
$F\left(e_{i}\right)$ and $\theta\left(F\left(e_{i}\right)\right)$, and shown as: $\left.\left.\quad \mathrm{C}=\left\{\left(F e_{i}\right) \mid \theta\left(\mathrm{F} e_{i}\right)\right)\right)\right\}, \forall e_{i} \in C$, where $\theta\left(F\left(e_{i}\right)\right)$ indicates the evaluator's confidence in his assessment of an alternative against $e_{i}$, and $\Theta=\left\{\theta\left(F\left(e_{i}\right)\right)\right\}, \forall e_{i} \in C ; F\left(e_{i}\right) \in$ $P(U) ; \theta\left(F\left(e_{i}\right)\right) \in[0,1]$. We denote each element of confidence information set as $S\left(e_{i}\right)=\left(F\left(e_{i}\right) \mid \theta\left(\mathrm{F}\left(e_{i}\right)\right)\right)$. Each element of the proposed CIS is a tuple of the agent's perceived value along with the agent's confidence in the same. This allows to consider the confidence degrees, often associated with the agent's perceived values in MCDM problems.

Example 3.1. Let the universal set $U=\left\{u_{1}, u_{2}, u_{3}, u_{4}\right\}$, where $u_{i}$ refers to as an alternative each of which is defined by the criteria $C=\left\{e_{1}, e_{2}\right\}$. The conventional information set is then shown as: $\mathcal{S}=\left\{S_{1}, S_{2}\right\}$, where $s_{i}$ refers to the information value corresponding to $e_{i}$. Let the confidence of the expert in his evaluation be $\theta\left(e_{1}\right)=(0.8)$ and $\theta\left(e_{2}\right)=(0.3)$. Then the corresponding confidence information set is $\mathrm{C}=$ $\left\{\left(S_{1} \mid 0.8\right),\left(S_{2} \mid 0.3\right)\right\}$. The confidence values are indicated in the bold, and are reflected in the final decision outcome. A few basic operations on the proposed CIS are presented as follows. Let $C^{1}$ and $C^{2}$ be two CISs defined over $(U, C)$. Then $C^{1}$ is a subset of $C^{2}$, i.e. $C^{1} \subseteq C_{2}$ iff i. $\theta\left(F^{1}\left(e_{i}\right)\right) \leq \theta\left(F^{2}\left(e_{i}\right)\right), \forall e_{i} \in C$; ii. $F^{1}\left(e_{i}\right) \subseteq F^{2}\left(e_{i}\right), \forall e_{i} \in C$. The complement of $\mathcal{C}$, denoted by, $\bar{C}$ is defined as $\left.\bar{C}=\left\{\bar{S}_{l} \mid \bar{\theta}_{\iota}=1-\theta_{i}\right)\right\}, \forall e_{i} \in$ $C$ (13). The complement of a CIS can be seen as an evaluation, just opposite to that of the given CIS. The union of $C_{1}$ and $C_{2}$, denoted by $C^{1} \cup C^{2}$, is defined as $\left.C^{1} \cup C^{2}=\left\{S_{i}{ }^{1} \cup s_{i}{ }^{2} \mid \theta_{i}{ }^{1} \cup \theta_{i}{ }^{2}\right)\right\}, \forall e_{i} \in C$. The intersection of $C^{1}$ and $C^{2}$ is: $\left.C^{1} \cap C^{2}=\left\{S_{i}{ }^{1} \cap s_{i}{ }^{2} \mid \theta_{i}{ }^{1} \cap \theta_{i}{ }^{2}\right)\right\}, \forall e_{i} \in C$. The union and intersection of $\mathrm{C}_{\text {and }} \mathrm{C}_{1}$ is :

$$
\left.\left.\mathcal{C} \cup C^{1}=\left\{S_{1} \cup S_{1}{ }^{1} \mid \mathbf{0 . 8}\right),\left(S_{2} \cup S_{2}{ }^{1} \mid \mathbf{0 . 3}\right)\right\} ; C \cap \mathrm{C}^{1}=\left\{S_{1} \cap S_{1}{ }^{1} \mid \mathbf{0 . 1}\right),\left(S_{2} \cap S_{2}{ }^{1} \mid \mathbf{0 . 2}\right)\right\}
$$

3.1 Properties

Let $\mathrm{C}$ be a CIS defined over $(\mathrm{U}, \mathrm{C})$, then
i) $\mathrm{C} \subseteq(\mathrm{C} \cup \mathrm{C})$
ii) $(C \cap C) \subseteq C$
iii) $\mathrm{C} \cup \mathrm{C}_{\mathrm{O}}=\mathrm{C} \cap \mathrm{C}_{\mathrm{O}}=\mathrm{C}_{\mathrm{O}}$
iv) $\mathrm{C} \cup \mathrm{C}_{1}=\mathrm{C} \cap \mathrm{C}_{1}=\mathrm{C}$

\section{CONCLUSION}

The proposed confidence information set (CIS) provides a convenient data structure to represent the perceived values for an information source value, along with the corresponding confidence value. It is inspired from the fact that an agent often faces confusion, or lack of a complete confidence, in the assessment of imprecise values. The proposed structure provides a useful provision for the agent(s) to provide this crucial information to qualify the evaluations. In contrast to a hesitant fuzzy set, it is easier for the agent to give his/her degree of confidence for the evaluations given by him/her. Besides, the proposed CIS are compact and easier to operate with, in comparison to a hesitant information set. CIS finds a good potential in multi criteria decision making in providing evaluations qualified by their associated degrees of confidence. As a future work, the arithmetic and geometric aggregation operators for CIS can be explored, which can add to their usefulness as a datastructure in MCDM. Besides, it can also be extended to the domains of type-2, rough sets, ignorance functions, and intuitionistic fuzzy sets, to present multi-faceted uncertainty. CIS can also be conveniently fitted with learning systems such as a neural network.

\section{REFERENCES}

Aggarwal M. (2019): Hesitant information sets and application in group decision making. Appl. Soft Compu., 75, $120-129$.

Aggarwal M. (2016), and Hanmandlu, M.: Representing Uncertainty With Information Sets. IEEE Trans. on Fuzzy Systems, 24(1), 1-15.

Hanmandlu M. (2011) and Das, A.: Content-based Image Retrieval by Information Theoretic Measure. Defence Science Journal, 61(5), 415-430.

Zadeh L. A. (1965): Fuzzy sets. Information and Control, 8 (3), 338-353.

Zimmerman H. (1983). J.: Using fuzzy sets in operational research. European Journal of Operational Research, 13(3), 201-216. 